\title{
Correction: 2013 Online-only article citations
}

Cite as: Can Urol Assoc J 2013;7(5-6):e457. http://dx.doi.org/10.5489/cuaj.1397

Published online June 12, 2013.

C hers lecteurs et chères lectrices, en 2013, le Journal de I'Association des urologues du Canada (JAUC) a commencé à paraître chaque mois dans le but d'éliminer le retard accumulé dans la publication des articles soumis et de favoriser la diffusion rapide de la science. Nos numéros imprimés continueront à être livrés en février, avril, juin, août, octobre et décembre. Quant à nos articles en ligne exclusivement, ils seront mis à votre disposition les autres mois de l'année, soit en janvier, mars, mai, juillet, septembre et novembre.

À partir de 2013, la numérotation des publications consistera en deux chiffres (1-2, 3-4, etc.). La date de publication pour les versions tant imprimées qu'électroniques sera une association de deux mois (janvier-février, mars-avril, etc.) De plus, les articles en ligne exclusivement (dont la pagination est précédée d'un E) comporteront la mention : Publié en ligne le [date].

Nous aimerions également porter à votre attention le fait que nous avons dû rectifier la numérotation des 13 premiers articles mis en ligne en 2013. La façon dont ceux-ci étaient répartis posait problème dans les numéros 1-2 (soit janvier-février) et 3-4 (mars-avril). Ainsi, la numérotation a été corrigée de façon à ce que ces articles soient associés au bon numéro du Journal.

\section{Issue 7(1-2)}

1: Imbeault A, Bernard G, Rousseau A, Morissette A, Chabaud S, Bouhout S, Bolduc S. An endothelialized urothelial cell-seeded tubular graft for urethral replacement. Can Urol Assoc J. 2013 Jan;7(1-2):E4-9. doi: 10.5489/cuaj.12217. PubMed PMID: 23401738; PubMed Central PMCID: PMC3559612.

2: Kapoor A, Touma NJ, Dib RE. Review of the efficacy and safety of cryoablation for the treatment of small renal masses. Can Urol Assoc J. 2013 Jan;7(1-2):E38-44. doi: 10.5489/cuaj.12018. PubMed PMID: 23401737; PubMed Central PMCID: PMC3559618.

3: Dekou A, Konan PG, Yao B, Vodi C, Fofana A, Manzan K. Éléphantiasis pénoscrotal idiopathique : une nouvelle observation et revue de la littérature. Can Urol Assoc J. 2013 Jan;7(1-2):E29-E32. French. PubMed PMID: 23401736; PubMed Central PMCID: PMC3559616.
4: Levine MA, Evans H. Open removal as a first-line treatment of magnetic intravesical foreign bodies. Can Urol Assoc J. 2013 Jan;7(1-2):E25-8. doi: 10.5489/cuaj.12043. PubMed PMID: 23401735; PubMed Central PMCID: PMC3559615.

5: Nason GJ, Tareen F, Mortell A. The top 100 cited articles in urology: An update. Can Urol Assoc J. 2013 Jan;7(1-2):E16-24. doi: 10.5489/cuaj.12223. PubMed PMID: 23401734; PubMed Central PMCID: PMC3559614.

6: Welk B, Kodama R, Macneily A. The newly graduated Canadian urologist: Over-trained and underemployed? Can Urol Assoc J. 2013 Jan;7(1-2):E10-5. doi: 10.5489/cuaj.12095. PubMed PMID: 23401733; PubMed Central PMCID: PMC3559613.

7: Anand A, Tullis E, Stephenson A, Nickel IC, Leveridge MJ. Pseudomonas aeruginosa bacteremia and prostatitis in a patient with cystic fibrosis. Can Urol Assoc J. 2013 Jan;7(1-2):E1-3. doi: 10.5489/ cuaj.11172. PubMed PMID: 23401732; PubMed Central PMCID: PMC3559611.

\section{3; Issue 7(3-4)}

1: Ziouziou I, Rhannam Y, Yacoubi S, Karmouni T, El Khader K, Koutani A, Andaloussi Al, Nouri A, Barre P, Nouri M. [Pudendal neuralgia: Report on three cases]. Can Urol Assoc J. 2013 Mar;7(34):E180-2. doi: 10.5489/cuaj.489. French. PubMed PMID: 23589754; PubMed Central PMCID: PMC3612407.

2: Mullins JK, Rodriguez R. Renal cell carcinoma seeding of a percutaneous biopsy tract. Can Urol Assoc J. 2013 Mar;7(3-4):E176-9. doi: 10.5489/cuaj.499. PubMed PMID: 23589753; PubMed Central PMCID: PMC3612408.

3: Haggag YM, Morsy G, Badr MM, Al Emam AB, Farid M, Etafy M. Comparative study of laparoscopic pyelolithotomy versus percutaneous nephrolithotomy in the management of large renal pelvic stones. Can Urol Assoc J. 2013 Mar;7 (3-4):E171-5. doi: 10.5489/cuaj.490. PubMed PMID: 23589752; PubMed Central PMCID: PMC3612403.

4: Amit A, Arun K, Bharat B, Navin R, Sameer T, Shankar DU. Penile fracture and associated urethral injury: Experience at a tertiary care hospital. Can Urol Assoc J. 2013 Mar;7(3-4):E168-70. doi: 10.5489/cuaj.475. PubMed PMID: 23589751; PubMed Central PMCID: PMC3612405.

5: Monga N, Sayani A, Rubinger DA, Wilson TH, Su Z. The effect of dutasteride on the detection of prostate cancer: A set of meta-analyses. Can Urol Assoc J. 2013 Mar;7(3-4):E161-7. doi: 10.5489/ cuaj.477. PubMed PMID: 23589750; PubMed Central PMCID: PMC3612406.

6: Kim TB, Lee SC, Kim KH, Jung H, Yoon SJ, Oh JK. The feasibility of shockwave lithotripsy for treating solitary, lower calyceal stones over $1 \mathrm{~cm}$ in size. Can Urol Assoc J. 2013 Mar;7 (3-4):E156-60. doi: 10.5489/cuaj.473. PubMed PMID: 23589749; PubMed Central PMCID: PMC3612404. 